\title{
Existence of financial equilibria with endogenous short selling restrictions and real assets
}

\author{
Michele Gori \\ Dipartimento di Matematica per le Decisioni, \\ Università degli Studi di Firenze \\ via delle Pandette 9, 50127 Firenze \\ e-mail: michele.gori@unifi.it \\ Marina Pireddu \\ Department of Economics, \\ European University Institute \\ via della Piazzuola 43, 50133 Firenze \\ e-mail: Marina.Pireddu@EUI.eu \\ Antonio Villanacci \\ Dipartimento di Matematica per le Decisioni, \\ Università degli Studi di Firenze \\ via delle Pandette 9, 50127 Firenze \\ e-mail: antonio.villanacci@unifi.it
}

August 24, 2012

\begin{abstract}
We consider a model with real assets and restricted participation described by household specific price dependent short selling constraints. We show existence of equilibria for all elements in an explicitly characterized large subset of the set of economies.
\end{abstract}

Keywords: General equilibrium; Restricted participation; Financial markets; Real assets.

JEL classification: D50, D52, D53.

\section{Introduction}

We analyze a general financial equilibrium model with real assets and restricted participation. Assets are said to be real if they promise to deliver bundles of commodities. Participation is said to be restricted if each asset demand has to belong to a household specific set.

For a recent, brief survey of the literature on restricted participation see Carosi et al. (2009). The standard main reference on financial equilibrium with real assets is still Duffie and Shafer (1985). As it is well known, in the case of incomplete markets with real assets, equilibria may not exist since changes in prices may change the rank of the return matrix causing a discontinuity in the demand function. On the other hand, using a description of the financial structure in terms of Grassmannian manifolds, Duffie and Shafer (1985) show existence for every utility function vector, and every endowment and yield vector in an open and full measure set.

There are only few papers which combine real assets and restricted participation in a sufficiently general manner and most of them deal with the context of default and collateral. Radner (1972) proves existence of equilibria for a modification of the general equilibrium model with incomplete financial markets, in which there is an exogenous upper bound on the short sale of assets. Geanakoplos (1997) provides an existence result for a model with default and durable goods in which the requirement that security sales 
are collateralized places an endogenous bound on short sales. Araujo et al. (2000) consider the model in Geanakoplos (1997), but with endogenous collateral and a continuum of households. Some assumptions are made along the paper so that consumers' short sales are uniformly bounded in order to meet the firstperiod budget constraint ${ }^{1}$. Dubey et al. (2005) consider a model with default in which the punishment for insolvent households consists in a decrease of their utility function. Existence of equilibria is proven when short sales are exogenously bounded. Bisin et al. (2010) present a model in which consumers have discretion over deliveries on contracts and a pooling mechanism is present. Some commodities are not subject to resale and for these commodities deliveries can only be made out of individuals' initial endowments. An explicit assumption on compactness for both deliveries and sales of all contracts except one is made in order to prove an existence result. Seghir and Torres-Martinez (2011) prove existence of equilibria for a model with financial structures including real assets and restrictions on borrowing which concern only the demand of (possibly) durable goods. Such context is general enough to encompass the framework in Geanakoplos (1997). In all above quoted papers, participation constraints are either exogenous or depend on some endogenous variables different from prices. We believe however that the access to the credit market can hardly be assumed to be constant in different inflation regimes.

In the present work, we thus combine the presence of real assets with simple, economically sound participation restrictions on financial markets. More precisely, each household faces an asset specific short selling constraint which depends upon prices, the existence of exogenously given bounds being quite hard to justify on economic ground. The dependence of financial restrictions on prices is shared with the paper by Carosi et al. (2009). In fact, the two papers bear some resemblances as well as crucial differences. In both cases the framework is in fact given by the classical pure exchange, general equilibrium model, with two periods and a finite number of states, commodities, assets, and households, with the additional constraint that asset demand is restricted to belong to a household specific portfolio set. In the paper by Carosi et al. (2009) however only numeraire assets are considered and the class of restrictions therein does not include our short selling constraints. In fact the present specification of portfolio sets implies, in particular, that the participation in the asset markets of each household depends on her demand of each asset, while in Carosi et al. (2009) it is instead required that, for each asset, there is a household whose participation does not depend on the demand of that asset.

Except for the crucial feature of endogeneity of the constraints, our model shows at first sight a strong similarity also with the paper by Radner (1972). When looking more closely at that work, the definition of equilibrium therein seems however to be too restrictive, as it includes a so called "free disposal" condition, meaning that at each state every household does "not plan to deliver more than he would have available from his resources after subtracting his consumption". Such condition plays a crucial role in the proof of existence, which, as the author says, turns out to be very similar to the one in Debreu (1959) and therefore it is only sketched. On the other hand, the endogeneity of our restrictions and the more general definition of equilibrium we consider imply that a proof is needed.

Our existence proof is based upon a homotopy method. A key ingredient in that method is to find a well chosen economy to build the needed homotopy function. In many general equilibrium models, that goal is accomplished using an arbitrary Pareto optimal allocation. With real assets and restricted participation, that property is not enough: indeed, the allocation has to have the additional property of being associated with supporting prices at which the return matrix has full rank. The set $\mathcal{E}^{\diamond}$ of economies for which such Pareto optimal allocations exist is then a subset of the set of economies for which an equilibrium itself exists $^{2}$. Observe that it is the use of the homotopy approach which guides in the identification of that subset.

Conditions on yields which ensure that the associated economies belong to $\mathcal{E}^{\diamond}$ are then presented. Indeed, we show existence of equilibria for all economies described by strictly positive endowments, standard smooth utility functions, general short selling constraints and assets that are of the numeraire type, or assets which deliver general bundles of commodities, as long as yield vectors are sufficiently diversified in each state. Further, when the number of assets does not exceed the number of commodities, the set of economies we determine surely contains an open and dense subset of the whole set of economies.

We finally observe that, at least in the framework we are dealing with, the approach we use seems to have some advantage with respect to the Grassmannian one by Duffie and Shafer (1985). Indeed, the

\footnotetext{
${ }^{1}$ Both in the framework in Geanakoplos (1997) and Araujo et al. (2000), the models have to be rewritten in an equivalent manner in order to make the presence of restricted participation more explicit, as done in Seghir and Torres-Martinez (2011).

${ }^{2}$ See (11) and Theorem 2.
} 
latter one, which is commonly used when working with real assets (see, for instance, Geanakoplos and Shafer (1990), Hirsch et al. (1990), Husseini et al. (1990) and Bottazzi (1995)), has always led only to generic existence result: given an economy, on the basis of those results, nothing can be concluded about the existence of a equilibrium for that economy.

In the remainder of the paper, we first describe the set-up of the model and the main results in Section 2 and we present proofs in Section 3.

\section{Set-up of the model and main results}

Our model builds up on the very standard two-period, pure exchange economy with uncertainty and financial markets. We consider a commodity market in which $C \geq 2$ types of different commodities, denoted by $c \in \mathcal{C}=\{1,2, \ldots, C\}$, are traded both today and tomorrow. We assume that tomorrow only one among $S \geq 1$ possible states of the world, denoted by $s \in\{1, \ldots, S\}$, will occur. We denote today by $s=0$ and we define $\mathcal{S}=\{0,1, \ldots, S\}$. Asset markets open in the first period, and there are $A \geq 1$ assets traded, denoted by $a \in \mathcal{A}=\{1,2, \ldots, A\}$. We assume $A \leq S$. Finally, there are $H \geq 2$ households, denoted by $h \in \mathcal{H}=\{1,2, \ldots, H\}$. The time structure of the model is as follows: today, households exchange commodities and assets, and consumption takes place. Then, tomorrow, uncertainty is resolved, households honor their financial obligations, and they again exchange and consume commodities.

We denote by $x_{h}^{c}(s) \in \mathbb{R}_{++}$the consumption of commodity $c$ in state $s$ by household $h$ and by $e_{h}^{c}(s) \in \mathbb{R}_{++}$the endowment of commodity $c$ in state $s$ owned by household $h$. We define

$$
\begin{array}{ll}
x_{h}(s)=\left(x_{h}^{c}(s)\right)_{c \in \mathcal{C}} \in \mathbb{R}_{++}^{C}, & x_{h}=\left(x_{h}(s)\right)_{s \in \mathcal{S}} \in \mathbb{R}_{++}^{G}, \quad x=\left(x_{h}\right)_{h \in \mathcal{H}} \in \mathbb{R}_{++}^{G H}, \\
e_{h}(s)=\left(e_{h}^{c}(s)\right)_{c \in \mathcal{C}} \in \mathbb{R}_{++}^{C}, & e_{h}=\left(e_{h}(s)\right)_{s \in \mathcal{S}} \in \mathbb{R}_{++}^{G}, \quad e=\left(e_{h}\right)_{h \in \mathcal{H}} \in \mathbb{R}_{++}^{G H},
\end{array}
$$

where $G=C(S+1)$. Household $h$ 's preferences are represented by a utility function $u_{h}: \mathbb{R}_{++}^{G} \rightarrow \mathbb{R}$. As in most of the literature on smooth economies we assume that, for every $h \in \mathcal{H}$,

$$
\begin{aligned}
& u_{h} \in C^{2}\left(\mathbb{R}_{++}^{G}\right) ; \\
& \text { for every } x_{h} \in \mathbb{R}_{++}^{G}, D u_{h}\left(x_{h}\right) \gg 0 ; \\
& \text { for every } v \in \mathbb{R}^{G} \backslash\{0\} \text { and } x_{h} \in \mathbb{R}_{++}^{G}, D u_{h}\left(x_{h}\right) v=0 \text { implies } v D^{2} u_{h}\left(x_{h}\right) v<0 ; \\
& \text { for every } \underline{x}_{h} \in \mathbb{R}_{++}^{G},\left\{x_{h} \in \mathbb{R}_{++}^{G}: u_{h}\left(x_{h}\right) \geq u_{h}\left(\underline{x}_{h}\right)\right\} \text { is closed in the topology of } \mathbb{R}^{G} \text {. }
\end{aligned}
$$

Let us denote by $\mathcal{U}$ the set of vectors $u=\left(u_{h}\right)_{h \in \mathcal{H}}$ of such utility functions. We denote by $p^{c}(s) \in \mathbb{R}_{++}$ the price of commodity $c$ at spot $s$, by $q^{a} \in \mathbb{R}$ the price of asset $a$ and by $z_{h}^{a} \in \mathbb{R}$ the quantity of asset $a$ held by household $h$. Moreover we define

$$
\begin{array}{ll}
p(s)=\left(p^{c}(s)\right)_{c \in \mathcal{C}} \in \mathbb{R}_{++}^{C}, & p=(p(s))_{s \in \mathcal{S}} \in \mathbb{R}_{++}^{G}, \quad q=\left(q^{a}\right)_{a \in \mathcal{A}} \in \mathbb{R}^{A}, \\
z_{h}=\left(z_{h}^{a}\right)_{a \in \mathcal{A}} \in \mathbb{R}^{A}, & z=\left(z_{h}\right)_{h \in \mathcal{H}} \in \mathbb{R}^{A H} .
\end{array}
$$

We denote by $y^{a, c}(s) \in \mathbb{R}$ the units of commodity $c$ delivered by one unit of asset $a$ in state $s$ and we define

$$
y^{a}(s)=\left(y^{a, c}(s)\right)_{c \in \mathcal{C}} \in \mathbb{R}^{C}, \quad y(s)=\left(y^{a}(s)\right)_{a \in \mathcal{A}} \in \mathbb{R}^{C A}, \quad y=(y(s))_{s \in\{1, \ldots, S\}} \in \mathbb{R}^{C A S} .
$$

Note in particular that, in state $s$, asset $a$ promises to deliver a vector $y^{a}(s)$ of commodities. Define the return matrix function as follows

$$
\begin{gathered}
\mathcal{R}: \mathbb{R}_{++}^{G} \times \mathbb{R}^{C A S} \rightarrow \mathbb{M}(S, A) \\
(p, y) \mapsto\left[p(s) y^{a}(s)\right]_{s \in\{1, \ldots, S\}, a \in \mathcal{A}}=\left[\begin{array}{ccccc}
p(1) y^{1}(1) & \ldots & p(1) y^{a}(1) & \ldots & p(1) y^{A}(1) \\
\vdots & \ddots & \vdots & \ddots & \vdots \\
p(s) y^{1}(s) & \ldots & p(s) y^{a}(s) & \ldots & p(s) y^{A}(s) \\
\vdots & \ddots & \vdots & \ddots & \vdots \\
p(S) y^{1}(S) & \ldots & p(S) y^{a}(S) & \ldots & p(S) y^{A}(S)
\end{array}\right]
\end{gathered}
$$


where $\mathbb{M}(S, A)$ is the space of the $S \times A$ matrices with real elements.

Consistently with our restricted participation framework, we assume that each household $h$ has only partial access, in a personalized manner to the asset market. In particular we assume that each household cannot sell more than a fixed quantity, depending on commodity and asset prices, of each asset. More precisely, we assume that, for every $h \in \mathcal{H}$, there is a function

$$
\sigma_{h}: \mathbb{R}_{++}^{G} \times \mathbb{R}^{A} \rightarrow \mathbb{R}^{A}, \quad(p, q) \mapsto\left(\sigma_{h}^{a}(p, q)\right)_{a \in \mathcal{A}}
$$

such that, for every $(p, q) \in \mathbb{R}_{++}^{G} \times \mathbb{R}^{A}, \sigma_{h}^{a}(p, q)$ represents the largest quantity of asset $a$ that household $h$ can sell at prices $(p, q)$ using asset $a$. We then call each function $\sigma_{h}$ short selling function. We assume that,

$$
\begin{aligned}
& \text { for every } h \in \mathcal{H}, \sigma_{h} \in C^{2}\left(\mathbb{R}_{++}^{G} \times \mathbb{R}^{A}, \mathbb{R}^{A}\right) ; \\
& \text { for every } h \in \mathcal{H}, a \in \mathcal{A},(p, q) \in \mathbb{R}_{++}^{G} \times \mathbb{R}^{A}, \sigma_{h}^{a}(p, q) \geq 0 ; \\
& \text { for every } a \in \mathcal{A},(p, q) \in \mathbb{R}_{++}^{G} \times \mathbb{R}^{A}, \sum_{h=1}^{H} \sigma_{h}^{a}(p, q)>0 .
\end{aligned}
$$

Let us denote by $\Sigma$ the set of vectors $\sigma=\left(\sigma_{h}\right)_{h \in \mathcal{H}}$ of such functions. The meaning of the above properties is simply described. Assumption (5) allows to use differential techniques. Assumption (6) permits no participation on financial markets and together with Assumption (7) allows each asset to be nontrivially exchanged in equilibrium.

We define the set of economies as

$$
\mathcal{E}=\mathbb{R}_{++}^{G H} \times \mathcal{U} \times \mathbb{R}^{C A S} \times \Sigma,
$$

with generic element $E=(e, u, y, \sigma)$, and for given $(p, q, E) \in \mathbb{R}_{++}^{G} \times \mathbb{R}^{A} \times \mathcal{E}$, we assume household $h \in \mathcal{H}$ has to solve the following maximization problem

$$
\begin{gathered}
\max _{\left(x_{h}, z_{h}\right)} u_{h}\left(x_{h}\right) \quad \text { s.t. } \\
\left\{\begin{array}{l}
p(0) x_{h}(0)+q z_{h}=p(0) e_{h}(0) \\
p(s) x_{h}(s)=p(s) e_{h}(s)+\left(p(s) y^{a}(s)\right)_{a=1}^{A} z_{h}, \quad s \in\{1, \ldots, S\} \\
z_{h}^{a}+\sigma_{h}^{a}(p, q) \geq 0
\end{array}\right.
\end{gathered}
$$

We are now ready to give the definition of equilibrium we use.

Definition 1. $\theta=\left(\left(x_{h}, z_{h}\right)_{h \in \mathcal{H}}, p, q\right) \in \mathbb{R}_{++}^{G H} \times \mathbb{R}^{A H} \times \mathbb{R}_{++}^{G} \times \mathbb{R}^{A}=\Theta$ is an equilibrium for $E \in \mathcal{E}$ if, for every $h \in \mathcal{H},\left(x_{h}, z_{h}\right)$ solves Problem $(8)$ at $(p, q, E)$ and $(x, z)$ satisfies market clearing conditions, that is,

$$
\sum_{h=1}^{H}\left(x_{h}-e_{h}\right)=0 \quad \text { and } \quad \sum_{h=1}^{H} z_{h}=0 .
$$

We denote by $\Theta(E)$ the set of equilibria for $E$ and we set

$$
\Theta_{\mathrm{n}}(E)=\left\{\theta \in \Theta(E): \forall s \in \mathcal{S}, p^{C}(s)=1\right\} .
$$

As mentioned in the Introduction, although our framework looks quite similar to the one in Carosi et al. (2009), the portfolio constraints we are dealing with are not a particular case of those considered in that paper. In fact, Carosi et al. (2009) fix an integer $J \geq 1$ and, for every $h \in \mathcal{H}$, assume there exists a function $r_{h}: \mathbb{R}^{A} \times \mathbb{R}_{++}^{G} \times \mathbb{R}^{A} \rightarrow \mathbb{R}^{J}$ such that the portfolio constraints of household $h$ are described by the inequality $r_{h}\left(z_{h}, p, q\right) \geq 0$. Such functions are called restriction functions by those authors. They further assume certain properties for the vector $r=\left(r_{h}\right)_{h \in \mathcal{H}}$, among which the following one

for every $a \in \mathcal{A}$ there exists $h \in \mathcal{H}$ such that,

$$
\text { for every }\left(z_{h}, p, q\right) \in \mathbb{R}^{A} \times \mathbb{R}_{++}^{G} \times \mathbb{R}^{A}, D_{z_{h}^{a}} r_{h}\left(z_{h}, p, q\right)=0 \text {, }
$$


which states that, for every asset, there is a household whose restriction function does not depend on the demand of that asset. Of course, our portfolio constraints can be simply written according to their notation assuming $J=A$ and setting, for every $h \in \mathcal{H}$ and for every $\left(z_{h}, p, q\right) \in \mathbb{R}^{A} \times \mathbb{R}_{++}^{G} \times \mathbb{R}^{A}$,

$$
r_{h}\left(z_{h}, p, q\right)=\left(z_{h}^{a}+\sigma_{h}^{a}(p, q)\right)_{a \in \mathcal{A}}
$$

However, it is immediate to check that, for every $\sigma \in \Sigma$, the corresponding vector $r$ never satisfies (10). We emphasize also that, differently from the model in Carosi et al. (2009), the presence of real assets in place of numeraire assets implies that the use of a homotopy method to prove existence is not standard but presents some difficulties requiring a more subtle argument.

In order to state the main existence theorem we need some definitions. We say that the allocation $\left(x_{h}\right)_{h \in \mathcal{H}} \in \mathbb{R}_{++}^{G H}$ is Pareto Optimal for $u \in \mathcal{U}$ if there is no allocation $\left(\widetilde{x}_{h}\right)_{h \in \mathcal{H}} \in \mathbb{R}_{++}^{G H}$ such that

$$
\sum_{h=1}^{H} \widetilde{x}_{h} \leq \sum_{h=1}^{H} x_{h} \quad \text { and } \quad\left(u_{h}\left(\widetilde{x}_{h}\right)\right)_{h \in \mathcal{H}}>\left(u_{h}\left(x_{h}\right)\right)_{h \in \mathcal{H}} .
$$

Denote the set of Pareto Optimal allocations for $u \in \mathcal{U}$ by $\mathcal{P}(u) \subseteq \mathbb{R}_{++}^{G H}$ and define the set

$$
\mathcal{E}^{\diamond}=\left\{(e, u, y, \sigma) \in \mathcal{E}: \exists x \in \mathcal{P}(u) \text { such that } \operatorname{rank}\left(\mathcal{R}\left(D u_{1}\left(x_{1}\right), y\right)\right)=A\right\}
$$

Theorem 2. For every $E \in \mathcal{E}^{\diamond}, \Theta_{\mathrm{n}}(E) \neq \varnothing$.

Conditions on yields which ensure that the associated economies belong to $\mathcal{E}^{\diamond}$ are presented below.

We say that $y \in \mathbb{R}^{C A S}$ belongs to $\mathcal{Y}_{1}$ if there exists $\mathcal{K} \subseteq\{1, \ldots, S\}$, with $^{3}|\mathcal{K}|=A$, and a function $\alpha:\{1 \ldots, S\} \rightarrow \mathcal{C}$ such that

$$
\forall(s, a, c) \in \mathcal{K} \times \mathcal{A} \times \mathcal{C}, c \neq \alpha(s) \Rightarrow y^{a, c}(s)=0,
$$

and

$$
\operatorname{rank}\left(\left[y^{a, \alpha(s)}(s)\right]_{s \in \mathcal{K}, a \in \mathcal{A}}\right)=A,
$$

while we say that $y \in \mathbb{R}^{C A S}$ belongs to $\mathcal{Y}_{2}$ if there exists $\mathcal{K} \subseteq\{1, \ldots, S\}$, with $|\mathcal{K}|=A$, such that

$$
\forall s \in \mathcal{K}, \operatorname{rank}\left(\left[y^{a, c}(s)\right]_{c \in \mathcal{C}, a \in \mathcal{A}}\right)=A .
$$

Yields in $\mathcal{Y}_{1}$ are associated with assets which deliver units of a unique, state dependent, commodity in each state in a subset of cardinality $A$ of all possible states. Yields of numeraire assets belong to $\mathcal{Y}_{1}$ (as long as the yield matrix has full rank): in that case, assets pay in units of the same commodity in each state. Yields in $\mathcal{Y}_{2}$ are associated with assets whose yield vectors are sufficiently diversified, in fact, linearly independent in $A$ states. For example, assumptions defining $\mathcal{Y}_{2}$ are satisfied if there are $A$ available commodities and in each state each asset $a$ delivers units of commodity $a$ only.

The following theorem explains the relationship between $\mathcal{Y}_{1}, \mathcal{Y}_{2}$ and $\mathcal{E}^{\diamond}$.

Theorem 3. $\mathbb{R}_{++}^{G H} \times \mathcal{U} \times\left(\mathcal{Y}_{1} \cup \mathcal{Y}_{2}\right) \times \Sigma \subseteq \mathcal{E}^{\diamond}$. Moreover, if $A \leq C$, then $\mathcal{Y}_{2}$ is an open and full measure subset of $\mathbb{R}^{C A S}$, otherwise $\mathcal{Y}_{2}=\varnothing$.

\section{Proof of the main results}

Throughout we are going to use the two following results (see Villanacci et al. (2002)).

Theorem 4. Let $m, p, n$ and $r$ be nonnegative integers, and let $M, \Omega$ and $N$ be $C^{r}$ manifolds of dimensions $m, p$ and $n$, respectively. Let $F: M \times \Omega \rightarrow N$ be a $C^{r}$ function. Assume $r>\max \{m-n, 0\}$. If $y$ is a regular value for $F$, then there exists a full measure subset $\Omega^{*}$ of $\Omega$ such that, for every $\omega \in \Omega^{*}$, $y$ is a regular value for $F_{\omega}: M \rightarrow N, x \mapsto F(x, \omega)$.

\footnotetext{
${ }^{3}$ Given a set $\mathcal{B}$, we denote its cardinality by $|\mathcal{B}|$.
} 
Theorem 5. Let $M, N$ be $C^{2}$ boundaryless manifolds of the same dimension, $y \in N$ and $F, G: M \rightarrow N$ be continuous functions. Assume that $G$ is $C^{1}$ in an open neighborhood $U$ of $G^{-1}(y)$, y is a regular value for $G$ restricted to $U,\left|G^{-1}(y)\right|$ is finite and odd, and there exists a continuous homotopy $H: M \times[0,1] \rightarrow N$ from $F$ to $G$ such that $H^{-1}(y)$ is compact. Then $F^{-1}(y) \neq \varnothing$.

Define the vectors

$$
\begin{aligned}
x_{h}^{\backslash}(s)=\left(x_{h}^{c}(s)\right)_{c \in\{1, \ldots, C-1\}} \in \mathbb{R}_{++}^{C-1}, & x_{h}^{\backslash}=\left(x_{h}^{\backslash}(s)\right)_{s \in \mathcal{S}} \in \mathbb{R}_{++}^{G-(S+1)}, \\
e_{h}^{\backslash}(s)=\left(e_{h}^{c}(s)\right)_{c \in\{1, \ldots, C-1\}} \in \mathbb{R}_{++}^{C-1}, & e_{h}^{\backslash}=\left(e_{h}^{\backslash}(s)\right)_{s \in \mathcal{S}} \in \mathbb{R}_{++}^{G-(S+1)} .
\end{aligned}
$$

As $S+1$ Walras' laws hold true in our model, the significant market clearing conditions in Definition 1 are in fact

$$
\sum_{h=1}^{H}\left(x_{h}^{\backslash}-e_{h}^{\backslash}\right)=0 \text { and } \sum_{h=1}^{H} z_{h}=0 .
$$

Since we are going to study equilibria in terms of first order conditions associated with households' maximization problems and (significant) market clearing conditions, define

$$
\Xi=\mathbb{R}_{++}^{G H} \times \mathbb{R}_{++}^{(S+1) H} \times \mathbb{R}^{A H} \times \mathbb{R}^{A H} \times \mathbb{R}_{++}^{G} \times \mathbb{R}^{A}
$$

with generic element

$$
\xi=\left(\left(x_{h}, \lambda_{h}, z_{h}, \mu_{h}\right)_{h \in \mathcal{H}}, p, q\right)=(x, \lambda, z, \mu, p, q)
$$

and the function

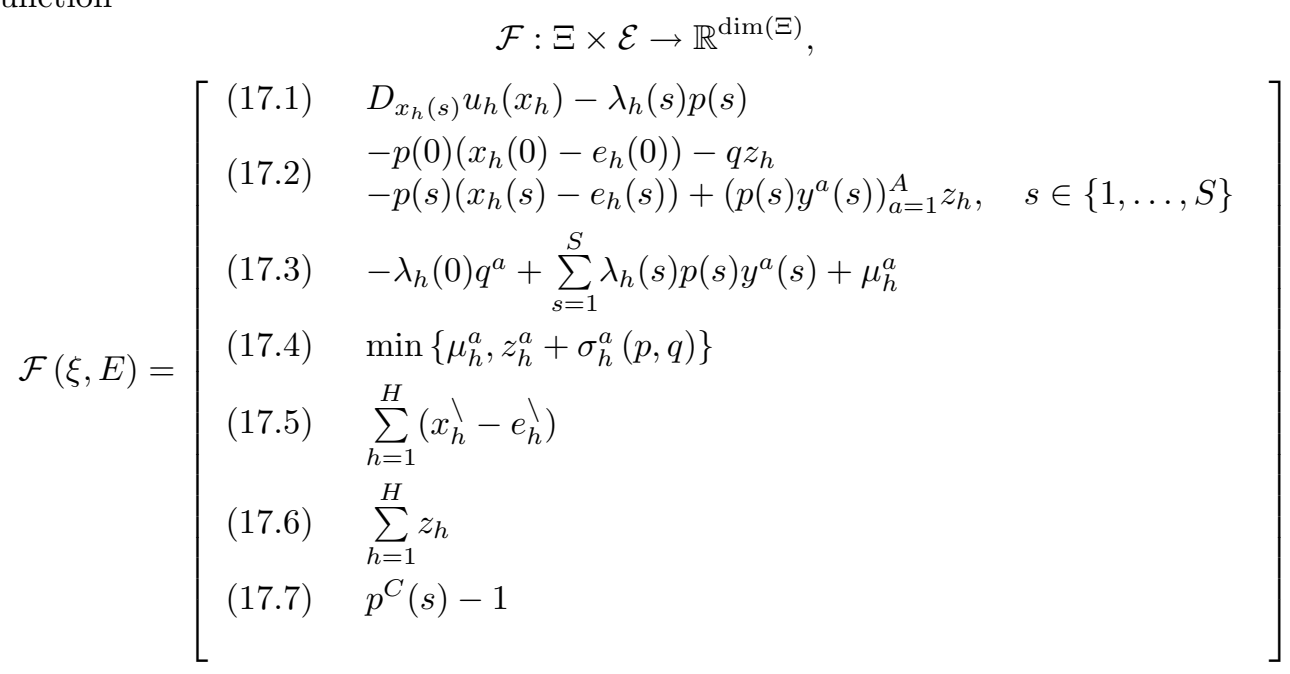

where $\operatorname{dim}(\Xi)$ denotes the dimension of the manifold (open set) $\Xi$.

Given now $E \in \mathcal{E}$, it is immediate to prove that if $\theta=\left(\left(x_{h}, z_{h}\right)_{h \in \mathcal{H}}, p, q\right) \in \Theta_{\mathrm{n}}(E)$, then there exists $\left(\lambda_{h}, \mu_{h}\right)_{h \in \mathcal{H}} \in \mathbb{R}_{++}^{(S+1) H} \times \mathbb{R}^{A H}$ such that $\xi=\left(\left(x_{h}, \lambda_{h}, z_{h}, \mu_{h}\right)_{h \in \mathcal{H}}, p, q\right) \in \Xi$ solves system $\mathcal{F}(\xi, E)=0$. Vice versa, if $\xi=\left(\left(x_{h}, \lambda_{h}, z_{h}, \mu_{h}\right)_{h \in \mathcal{H}}, p, q\right) \in \Xi$ solves system $\mathcal{F}(\xi, E)=0$, then $\left(\left(x_{h}, z_{h}\right)_{h \in \mathcal{H}}, p, q\right) \in$ $\Theta_{\mathrm{n}}(E)$.

Of course, Theorem 2 is a consequence of the following result, we prove below via Theorem 5 .

Theorem 6. For every $E \in \mathcal{E}^{\diamond}$, there exists $\xi \in \Xi$ such that $\mathcal{F}(\xi, E)=0$.

Proof. Let $E=(e, u, y, \sigma) \in \mathcal{E}^{\diamond}$ be given, and define

$$
F: \Xi \rightarrow \mathbb{R}^{\operatorname{dim}(\Xi)}, \quad \xi \mapsto \mathcal{F}(\xi, E) .
$$


Fix $\varepsilon>0$ and $x^{*} \in \mathcal{P}(u)$ such that $\operatorname{rank}\left(\mathcal{R}\left(D u_{1}\left(x_{1}^{*}\right), y\right)\right)=A$. Consider then the system in the unknowns $\xi=(x, \lambda, z, \mu, p, q) \in \Xi$ and $\tau \in[0,1]$, given by

$$
\begin{cases}(18.1) & D_{x_{h}(s)} u_{h}\left(x_{h}\right)-\lambda_{h}(s) p(s)=0 \\ (18.2) & -p(0)\left(x_{h}(0)-\left((1-\tau) e_{h}(0)+\tau x_{h}^{*}(0)\right)\right)-q z_{h}=0 \\ & -p(s)\left(x_{h}(s)-\left((1-\tau) e_{h}(s)+\tau x_{h}^{*}(s)\right)\right)+\left(p(s) y^{a}(s)\right)_{a=1}^{A} z_{h}=0, \quad s \in\{1, \ldots, S\} \\ (18.3) & -\lambda_{h}(0) q^{a}+\sum_{s=1}^{S} \lambda_{h}(s) p(s) y^{a}(s)+\mu_{h}^{a}=0 \\ (18.4) & \min \left\{\mu_{h}^{a}, z_{h}^{a}+(1-\tau) \sigma_{h}^{a}(p, q)+\tau \varepsilon\right\}=0 \\ (18.5) & \sum_{h=1}^{H}\left(x_{h}^{\backslash}-\left((1-\tau) e_{h}^{\backslash}+\tau x_{h}^{*}\right)\right)=0 \\ (18.6) & \sum_{h=1}^{H} z_{h}=0 \\ (18.7) & p^{C}(s)-1=0 \\ & \end{cases}
$$

and define the functions

$$
H: \Xi \times[0,1] \rightarrow \mathbb{R}^{\operatorname{dim}(\Xi)}, \quad(\xi, \tau) \mapsto \text { left hand side of System (18), }
$$

and

$$
G: \Xi \rightarrow \mathbb{R}^{\operatorname{dim}(\Xi)}, \quad \xi \mapsto H(\xi, 1)
$$

Observe that, for every $\xi \in \Xi, H(\xi, 0)=F(\xi)$, and that $F, H, G$ are $C^{0}$ in $\Xi$. Note also that, for every $(p, q, \tau)$ and $h \in \mathcal{H}$, if $\left(x_{h}, \lambda_{h}, z_{h}, \mu_{h}\right)$ solves equations (18.1)-(18.4), then $\left(x_{h}, z_{h}\right)$ is optimal solution to the maximization problem

$$
\begin{gathered}
\max _{\left(x_{h}, z_{h}\right)} u_{h}\left(x_{h}\right) \text { s.t. } \\
\left\{\begin{array}{l}
p(0) x_{h}(0)+q z_{h}=p(0)\left((1-\tau) e_{h}(0)+\tau x_{h}^{*}(0)\right) \\
p(s) x_{h}(s)=p(s)\left((1-\tau) e_{h}(s)+\tau x_{h}^{*}(s)\right)+\left(p(s) y^{a}(s)\right)_{a=1}^{A} z_{h}, \quad s \in\{1, \ldots, S\} \\
z_{h}^{a}+(1-\tau) \sigma_{h}^{a}(p, q)+\tau \varepsilon \geq 0
\end{array}\right.
\end{gathered}
$$

and, vice versa, if $\left(x_{h}, z_{h}\right)$ is optimal solution to (19), then there exists $\left(\lambda_{h}, \mu_{h}\right)$ such that $\left(x_{h}, \lambda_{h}, z_{h}, \mu_{h}\right)$ solves equations (18.1)-(18.4). Moreover, if $(\xi, \tau) \in \Xi \times[0,1]$ is such that $H(\xi, \tau)=0$, then indeed

$$
\sum_{h=1}^{H}\left(x_{h}-\left((1-\tau) e_{h}+\tau x_{h}^{*}\right)\right)=0 .
$$

Finally, if we prove that

$$
\begin{aligned}
& G^{-1}(0)=\left\{\xi^{*}\right\} \text { and } G \text { is } C^{1} \text { in an open neighborhood of } \xi^{*}, \\
& D_{\xi} G\left(\xi^{*}\right) \text { is not singular, } \\
& H^{-1}(0) \text { is compact, }
\end{aligned}
$$

for some $\xi^{*} \in \Xi$, then Theorem 5 can be applied to get $F^{-1}(0) \neq \varnothing$. In what follows, we are going to prove that conditions (21), (22) and (23) hold true.

In order to prove $(21)$, let us show that

$$
G^{-1}(0)=\left\{\xi^{*}\right\}=\left\{\left(\left(x_{h}^{*}, \lambda_{h}^{*}, z_{h}^{*}, \mu_{h}^{*}\right)_{h \in \mathcal{H}}, p^{*}, q^{*}\right)\right\},
$$

where $\left(x_{h}^{*}\right)_{h \in \mathcal{H}}$ is the element of $\mathcal{P}(u)$ used in the definition of System (18) and 


$$
\begin{aligned}
\forall h \in \mathcal{H}, \quad \lambda_{h}^{*} & =\left(\frac{D_{x_{h}^{C}(S)} u_{h}\left(x_{h}^{*}\right)}{D_{x_{1}^{C}(S)} u_{1}\left(x_{1}^{*}\right)} D_{x_{1}^{C}(s)} u_{1}\left(x_{1}^{*}\right)\right)_{s \in \mathcal{S}} \in \mathbb{R}_{++}^{S+1}, \\
\forall h \in \mathcal{H}, \quad z_{h}^{*} & =0 \in \mathbb{R}^{A}, \\
\forall h \in \mathcal{H}, \quad \mu_{h}^{*} & =0 \in \mathbb{R}^{A}, \\
p^{*} & =\left(\frac{D_{x_{1}(s)} u_{1}\left(x_{1}^{*}\right)}{D_{x_{1}^{C}(s) u_{1}\left(x_{1}^{*}\right)}^{*}}\right)_{s \in \mathcal{S}} \in \mathbb{R}_{++}^{G}, \\
q^{*} & =\left(\sum_{s=1}^{S} \frac{D_{x_{1}(s)} u_{1}\left(x_{1}^{*}\right)}{D_{x_{1}^{C}(0)} u_{1}\left(x_{1}^{*}\right)} y^{a}(s)\right)_{a \in \mathcal{A}} \in \mathbb{R}^{A} .
\end{aligned}
$$

Indeed, as $x^{*}=\left(x_{h}^{*}\right)_{h \in \mathcal{H}} \in \mathcal{P}(u)$, we know that there exists $\left(\theta^{*}, \gamma^{*}\right)=\left(\left(\theta_{h}^{*}\right)_{h \in \mathcal{H}}, \gamma^{*}\right) \in \mathbb{R}^{H} \times \mathbb{R}^{G}$ such that $\left(x^{*}, \theta^{*}, \gamma^{*}\right)$ solves the system

$$
\left\{\begin{array}{l}
\theta_{h} D u_{h}\left(x_{h}\right)-\gamma=0 \\
\theta_{1}-1=0 \\
\sum_{h=1}^{H}\left(x_{h}-x_{h}^{*}\right)=0
\end{array}\right.
$$

Then it is immediate to prove that $G\left(\xi^{*}\right)=0$, that is, $\xi^{*}$ solves the system

$$
\begin{cases}(26.1) & D_{x_{h}(s)} u_{h}\left(x_{h}\right)-\lambda_{h}(s) p(s)=0 \\ (26.2) & -p(0)\left(x_{h}(0)-x_{h}^{*}(0)\right)-q z_{h}=0 \\ & -p(s)\left(x_{h}(s)-x_{h}^{*}(s)\right)+\left(p(s) y^{a}(s)\right)_{a=1}^{A} z_{h}=0, \quad s \in\{1, \ldots, S\} \\ (26.3) & -\lambda_{h}(0) q^{a}+\sum_{s=1}^{S} \lambda_{h}(s) p(s) y^{a}(s)+\mu_{h}^{a}=0 \\ (26.4) & \min \left\{\mu_{h}^{a}, z_{h}^{a}+\varepsilon\right\}=0 \\ (26.5) & \sum_{h=1}^{H}\left(x_{h}^{\backslash}-x_{h}^{*}\right)=0 \\ (26.6) & \sum_{h=1}^{H} z_{h}=0 \\ (26.7) & p^{C}(s)-1=0 \\ & \end{cases}
$$

Consider now

$$
\xi^{* *}=\left(\left(x_{h}^{* *}, \lambda_{h}^{* *}, z_{h}^{* *}, \mu_{h}^{* *}\right)_{h \in \mathcal{H}}, p^{* *}, q^{* *}\right) \in \Xi
$$

such that $G\left(\xi^{* *}\right)=0$, and prove that $\xi^{* *}=\xi^{*}$. Let us show at first that $x^{* *}=x^{*}$. Suppose by contradiction $x^{* *} \neq x^{*}$ and consider $\widetilde{x}=\frac{1}{2}\left(x^{*}+x^{* *}\right)$. Since $G\left(\xi^{* *}\right)=0$, then

$$
\sum_{h=1}^{H} \widetilde{x}_{h}=\frac{1}{2} \sum_{h=1}^{H}\left(x_{h}^{*}+x_{h}^{* *}\right)=\sum_{h=1}^{H} x_{h}^{*} .
$$

Moreover we have also that, for every $h \in \mathcal{H},\left(x_{h}^{* *}, z_{h}^{* *}\right)$ is the maximum for Problem (19), considered at prices $p^{* *}$ and $q^{* *}$ and $\tau=1$. As $\left(x_{h}^{*}, z_{h}^{*}\right)$ is feasible for the same problem, we obtain $u_{h}\left(x_{h}^{* *}\right) \geq u_{h}\left(x_{h}^{*}\right)$ and then, by (3) and $x^{* *} \neq x^{*}$, it follows

$$
\left(u_{h}\left(\widetilde{x}_{h}\right)\right)_{h \in \mathcal{H}}>\left(u_{h}\left(x_{h}^{*}\right)\right)_{h \in \mathcal{H}} .
$$

Since (27) and (28) imply that $x^{*} \notin \mathcal{P}(u)$, the contradiction is finally found.

Let us prove now that $\lambda^{* *}=\lambda^{*}$. From (26.7) we know that, for every $s \in \mathcal{S}$,

$$
p^{* * C}(s)=1=p^{* C}(s),
$$

and then from (26.1) we find that, for every $h \in \mathcal{H}, s \in \mathcal{S}$,

$$
\lambda_{h}^{* *}(s)=D_{x_{h}^{C}(s)} u_{h}\left(x_{h}^{*}\right)=\lambda_{h}^{*}(s) .
$$


From the above relationship we immediately deduce $p^{* *}=p^{*}$, as $(26.1)$ implies that, for every $s \in \mathcal{S}$,

$$
p^{* *}(s)=\frac{D_{x_{h}(s)} u_{h}\left(x_{h}^{*}\right)}{\lambda_{h}^{*}(s)}=p^{*}(s) .
$$

In order to prove that $z^{* *}=z^{*}$, observe that by (11) the definition of $p^{*} \operatorname{implies} \operatorname{rank}\left(\mathcal{R}\left(p^{*}, y\right)\right)=$ A. Moreover, from (26.2) and the equalities $x^{* *}=x^{*}$ and $p^{* *}=p^{*}$, we have that, for every $h \in \mathcal{H}$, $\mathcal{R}\left(p^{*}, y\right) z_{h}^{* *}=0$, that is, $z_{h}^{* *}=0$. Then we get the equality $z^{* *}=z^{*}$.

Clearly, from (26.4), $\mu^{* *}=\mu^{*}=0$. Finally, from (26.3), we obtain that, for every $a \in \mathcal{A}, h \in \mathcal{H}$,

$$
q^{* * a}=\frac{1}{\lambda_{h}^{*}(0)}\left(\sum_{s=1}^{S} \lambda_{h}^{*}(s) p^{*}(s) y^{a}(s)+\mu_{h}^{* a}\right)=q^{* a} .
$$

and then $q^{* *}=q^{*}$, as well. Then we have obtained that $\xi^{* *}=\xi^{*}$ and, since it is immediate to show that $G$ is $C^{1}$ in an open neighborhood of $\xi^{*},(21)$ is proved.

The proof of (22) is indeed a very simple modification of the argument in Villanacci et al. (2002), Chapter 11, Lemma 18. More precisely, the computation of $D_{\xi} G\left(\xi^{*}\right)$ is described in the table below. Note that the components of the function $G$ are listed in the first column, the variables with respect to which derivatives are taken are listed in the first row, and in the remaining bottom right corner the Jacobian matrix is displayed.

\begin{tabular}{|c||c|c|c|c|c|c|}
\multicolumn{1}{c||}{} & $x_{h}$ & $\lambda_{h}$ & $z_{h}$ & $\mu_{h}$ & $p$ & $q$ \\
\hline \hline$(26.1)$ & $D^{2} u_{h}\left(x_{h}^{*}\right)$ & $-\Phi\left(p^{*}\right)^{T}$ & & & $-\Lambda_{h}^{*}$ & \\
\hline$(26.2)$ & $-\Phi\left(p^{*}\right)$ & & {$\left[\begin{array}{c}-q^{*} \\
\mathcal{R}\left(p^{*}, y\right)\end{array}\right]$} & & & \\
\hline$(26.3)$ & & {$\left[\begin{array}{c}-q^{*} \\
\mathcal{R}\left(p^{*}, y\right)\end{array}\right]^{T}$} & & $I_{A}$ & $Y_{h}^{*}$ & $-\lambda_{h}^{*}(0) I_{A}$ \\
\hline$(26.4)$ & & & & $I_{A}$ & & \\
\hline$(26.5)$ & $L$ & & & & & \\
\hline$(26.6)$ & & & $I_{A}$ & & & \\
\hline$(26.7)$ & & & & & $M$ & \\
\hline
\end{tabular}

where, for every positive integer $N, I_{N}$ is the identity matrix of dimension $N$, and

$$
\begin{aligned}
& \Phi(p)=\left[\begin{array}{llllllll}
p^{1}(0) & \cdots & p^{C-1}(0) & 1 & & & & \\
& & & & \ddots & & & \\
& & & & p^{1}(S) & \cdots & p^{C-1}(S) & 1
\end{array}\right]_{(S+1) \times G} \\
& M=\left[\begin{array}{lllllllll}
0 & \cdots & 0 & 1 & & & & & \\
& & & & \ddots & & & & \\
& & & & & 0 & \cdots & 0 & 1
\end{array}\right]_{(S+1) \times G} \\
& \Lambda_{h}^{*}=\left[\begin{array}{ccc}
\lambda_{h}^{*}(0) I_{C} & & \\
& \ddots & \\
& & \lambda_{h}^{*}(S) I_{C}
\end{array}\right]_{G \times G} \\
& L=\left[\begin{array}{ccccc}
I_{C-1} & 0 & & & \\
& & \ddots & & \\
& & & I_{C-1} & 0
\end{array}\right]_{\{G-(S+1)\} \times G} \\
& Y_{h}^{*}=\left[\begin{array}{cccc}
\mathbf{0} & \lambda_{h}^{*}(1) y^{1}(1) & \ldots & \lambda_{h}^{*}(S) y^{1}(S) \\
\vdots & \vdots & \ddots & \vdots \\
\mathbf{0} & \lambda_{h}^{*}(1) y^{A}(1) & \ldots & \lambda_{h}^{*}(S) y^{A}(S)
\end{array}\right]_{A \times G}
\end{aligned}
$$

with $\mathbf{0}=(0, \ldots, 0) \in \mathbb{R}^{C}$. In order to simplify our argument, we make some preliminary observations. Thanks to the presence of $I_{A}$ in correspondence to $\mu_{h}$ and the fact that there are no other nonnull elements 
on its super-row, we are left with proving it is not singular the matrix obtained from $D_{\xi} G\left(\xi^{*}\right)$ by erasing the super-row and the super-column corresponding to $\mu_{h}$. Moreover, with the nonnull elements of $M$ we can clean the corresponding columns and erase them, and finally erase the super-row of $M$, too. Define now

$$
\widetilde{\Lambda}_{h}^{*}=\left[\begin{array}{ccc}
\lambda_{h}^{*}(0) I_{C-1} & & \\
0 & & \\
& \ddots & \\
& & \lambda_{h}^{*}(S) I_{C-1} \\
& & 0
\end{array}\right]_{G \times\{G-(S+1)\}}
$$

and

$$
\widetilde{Y}_{h}^{*}=\left[\begin{array}{cccc}
\mathbf{0} & \lambda_{h}^{*}(1) y^{1 \backslash}(1) & \ldots & \lambda_{h}^{*}(S) y^{1 \backslash}(S) \\
\vdots & \vdots & \ddots & \vdots \\
\mathbf{0} & \lambda_{h}^{*}(1) y^{A \backslash}(1) & \ldots & \lambda_{h}^{*}(S) y^{A \backslash}(S)
\end{array}\right]_{A \times\{G-(S+1)\}}
$$

where $\mathbf{0} \backslash=(0, \ldots, 0) \in \mathbb{R}^{C-1}$ and $y^{a} \backslash(s)=\left(y^{a, c}(s)\right)_{c \in\{1, \ldots, C-1\}} \in \mathbb{R}^{C-1}$, for $a \in \mathcal{A}, s \in\{1, \ldots, S\}$. Introduce also $p \backslash=(p \backslash(s))_{s \in \mathcal{S}}$, with $p \backslash(s)=\left(p^{1}(s), \ldots, p^{C-1}(s)\right) \in \mathbb{R}_{++}^{C-1}$. Hence, we are led to prove that the following matrix, that we call $N\left(\xi^{*}\right)$, is not singular

\begin{tabular}{|c||c|c|c|c|c|}
\cline { 2 - 6 } \multicolumn{1}{c|}{} & $x_{h}$ & $\lambda_{h}$ & $z_{h}$ & $p^{\backslash}$ & $q$ \\
\hline \hline$(26.1)$ & $D^{2} u_{h}\left(x_{h}^{*}\right)$ & $-\Phi\left(p^{*}\right)^{T}$ & & $-\widetilde{\Lambda}_{h}^{*}$ & \\
\hline$(26.2)$ & $-\Phi\left(p^{*}\right)$ & & $\left.\begin{array}{c}-q^{*} \\
\mathcal{R}\left(p^{*}, y\right)\end{array}\right]$ & & \\
\hline$(26.3)$ & & {$\left[\begin{array}{c}-q^{*} \\
\mathcal{R}\left(p^{*}, y\right)\end{array}\right]^{T}$} & & $\widetilde{Y}_{h}^{*}$ & $-\lambda_{h}^{*}(0) I_{A}$ \\
\hline$(26.5)$ & $L$ & & & & \\
\hline$(26.6)$ & & & $I_{A}$ & & \\
\hline
\end{tabular}

Let us show that if $N\left(\xi^{*}\right) \Delta \zeta=0$, for some

$$
\Delta \zeta=\left(\left(\Delta x_{h}, \Delta \lambda_{h}, \Delta z_{h}\right)_{h \in \mathcal{H}}, \Delta p^{\backslash}, \Delta q\right) \in \mathbb{R}_{++}^{G H} \times \mathbb{R}_{++}^{(S+1) H} \times \mathbb{R}^{A H} \times \mathbb{R}_{++}^{G-(S+1)} \times \mathbb{R}^{A},
$$

then $\Delta \zeta=0$. We rewrite $N\left(\xi^{*}\right) \Delta \zeta=0$ as

$$
\begin{cases}(29.1) & D^{2} u_{h}\left(x_{h}^{*}\right) \Delta x_{h}-\Phi\left(p^{*}\right)^{T} \Delta \lambda_{h}-\widetilde{\Lambda}_{h}^{*} \Delta p \backslash=0 \\
(29.2) & -\Phi\left(p^{*}\right) \Delta x_{h}+\left[\begin{array}{c}
-q^{*} \\
\mathcal{R}\left(p^{*}, y\right)
\end{array}\right] \Delta z_{h}=0 \\
(29.3) & {\left[\begin{array}{c}
-q^{*} \\
\mathcal{R}\left(p^{*}, y\right)
\end{array}\right]^{T} \Delta \lambda_{h}+\left(\sum_{s=1}^{S} \lambda_{h}^{*}(s) y^{a} \backslash(s) \Delta p \backslash(s)\right)_{a \in \mathcal{A}}-\lambda_{h}^{*}(0) \Delta q=0} \\
(29.4) & \sum_{h=1}^{H} \Delta x_{h}^{\backslash}=0 \\
(29.5) & \sum_{h=1}^{H} \Delta z_{h}=0,\end{cases}
$$

where

$$
\begin{array}{ccc}
\Delta x_{h}^{\backslash}(s) & =\left(\Delta x_{h}^{c}(s)\right)_{c \in\{1, \ldots, C-1\}} \in \mathbb{R}_{++}^{C-1} \quad \text { and } \quad \Delta x_{h}^{\backslash}=\left(\Delta x_{h}^{\backslash}(s)\right)_{s \in \mathcal{S}} \in \mathbb{R}_{++}^{G-(S+1)}, \\
\Delta p^{\backslash}(s) & =\left(\Delta p^{c}(s)\right)_{c \in\{1, \ldots, C-1\}} \in \mathbb{R}_{++}^{C-1} \quad \text { and } & \Delta p^{\backslash}=\left(\Delta p^{\backslash}(s)\right)_{s \in \mathcal{S}} \in \mathbb{R}_{++}^{G-(S+1)} .
\end{array}
$$

Recall that, as $\left(x_{h}^{*}\right)_{h \in \mathcal{H}} \in \mathcal{P}(u)$, there exists $\left(\theta^{*}, \gamma^{*}\right) \in \mathbb{R}^{H} \times \mathbb{R}^{G}$ such that $\left(x^{*}, \theta^{*}, \gamma^{*}\right)$ solves System (25) 
(see Villanacci et al. (2002), Chapter 8, Lemma 39). Then, defined

$$
\Gamma^{*}=\left[\begin{array}{ccc}
\gamma^{* C}(0) I_{C-1} & & \\
0 & & \\
& \ddots & \\
& & \gamma^{* C}(S) I_{C-1} \\
& & 0
\end{array}\right]_{G \times G-(S+1)}
$$

where $\gamma^{*}=\left(\gamma^{* c}(s)\right)_{c \in \mathcal{C}, s \in \mathcal{S}}$, we have

$$
\widetilde{\Lambda}_{h}^{*}=\frac{1}{\theta_{h}^{*}} \Gamma^{*} .
$$

From (29.1), for every $h \in \mathcal{H}$, we have in particular

$$
\theta_{h}^{*} \Delta x_{h} D^{2} u_{h}\left(x_{h}^{*}\right) \Delta x_{h}-\theta_{h}^{*} \Delta x_{h} \Phi\left(p^{*}\right)^{T} \Delta \lambda_{h}-\theta_{h}^{*} \Delta x_{h} \widetilde{\Lambda}_{h}^{*} \Delta p \backslash=0,
$$

and from (29.2) and (30), we obtain

$$
\theta_{h}^{*} \Delta x_{h} D^{2} u_{h}\left(x_{h}^{*}\right) \Delta x_{h}=\theta_{h}^{*} \Delta z_{h}\left[\begin{array}{c}
-q^{*} \\
\mathcal{R}\left(p^{*}, y\right)
\end{array}\right]^{T} \Delta \lambda_{h}+\Delta x_{h} \Gamma^{*} \Delta p \backslash
$$

Using now (29.3) and observing that $\theta_{h}^{*} \lambda_{h}^{*}(s)=\gamma^{C *}(s), s \in \mathcal{S}$, we get the equality

$$
\theta_{h}^{*} \Delta x_{h} D^{2} u_{h}\left(x_{h}^{*}\right) \Delta x_{h}=\gamma^{C *}(0) \Delta z_{h} \Delta q-\Delta z_{h}\left(\sum_{s=1}^{S} \gamma^{C *}(s) y^{a \backslash}(s) \Delta p \backslash(s)\right)_{a \in \mathcal{A}}+\Delta x_{h} \Gamma^{*} \Delta p \backslash
$$

and summing up over $h \in \mathcal{H}$ and using (29.4) and (29.5), we obtain

$$
\sum_{h=1}^{H} \theta_{h}^{*} \Delta x_{h} D^{2} u_{h}\left(x_{h}^{*}\right) \Delta x_{h}=0 .
$$

Observe also that, for every $h \in \mathcal{H}$,

$$
D u_{h}\left(x_{h}^{*}\right) \Delta x_{h}=0 .
$$

Indeed, from (26.1), we obtain $D u_{h}\left(x_{h}^{*}\right) \Delta x_{h}=\lambda_{h}^{*} \Phi\left(p^{*}\right) \Delta x_{h}$, and then from (29.2), we get

$$
\lambda_{h}^{*} \Phi\left(p^{*}\right) \Delta x_{h}=\lambda_{h}^{*}\left[\begin{array}{c}
-q^{*} \\
\mathcal{R}\left(p^{*}, y\right)
\end{array}\right] \Delta z_{h}=-\mu_{h}^{*} \Delta z_{h}=0
$$

where the last two equalities follow by $(26.3)$ and $\mu_{h}^{*}=0$, respectively. Define now the set

$$
\mathcal{H}^{*}=\left\{h \in \mathcal{H}: \Delta x_{h} \neq 0\right\},
$$

and assume by contradiction $\mathcal{H}^{*} \neq \varnothing$. Since $\theta^{*} \in \mathbb{R}_{++}^{H}$, we have that (3) and (32) imply

$$
\sum_{h=1}^{H} \theta_{h}^{*} \Delta x_{h} D^{2} u_{h}\left(x_{h}^{*}\right) \Delta x_{h}=\sum_{h \in \mathcal{H}^{*}} \theta_{h}^{*} \Delta x_{h} D^{2} u_{h}\left(x_{h}^{*}\right) \Delta x_{h}<0 .
$$

As (33) contradicts (31), we get $\mathcal{H}^{*}=\varnothing$, that is, for every $h \in \mathcal{H}, \Delta x_{h}=0$. Note also that, from (29.2) and since $\mathcal{R}\left(p^{*}, y\right)$ has full rank, we immediately obtain that, for every $h \in \mathcal{H}, \Delta z_{h}=0$.

Let us show now that, for every $h \in \mathcal{H}, \Delta \lambda_{h}=0$. From (29.1), we know that, for every $h \in \mathcal{H}$,

$$
-\Phi\left(p^{*}\right)^{T} \Delta \lambda_{h}-\widetilde{\Lambda}_{h}^{*} \Delta p \backslash=0 .
$$

Then, for every $h \in \mathcal{H}, s \in \mathcal{S}$, we have $-p^{* C}(s) \Delta \lambda_{h}(s)=0$ and hence $\Delta \lambda_{h}(s)=0$, as desired.

Finally, as, for every $h \in \mathcal{H}, \Delta x_{h}=0$ and $\Delta \lambda_{h}=0$, from (29.1) and (29.3), we immediately obtain $\Delta p \backslash=0$ and $\Delta q=0$, respectively. Then $\Delta \zeta=0$ and the proof of (22) is complete. 
In order to show (23), we show that each sequence

$$
\left(\left(x_{h}^{[n]}, \lambda_{h}^{[n]}, z_{h}^{[n]}, \mu_{h}^{[n]}\right)_{h \in \mathcal{H}}, p^{[n]}, q^{[n]}, \tau^{[n]}\right)_{n \in \mathbb{N}}=\left(x^{[n]}, \lambda^{[n]}, z^{[n]}, \mu^{[n]}, p^{[n]}, q^{[n]}, \tau^{[n]}\right)_{n \in \mathbb{N}}
$$

in $H^{-1}(0)$ admits a converging subsequence. As we are going to use a diagonal argument, every time we say that a sequence converges we mean it has a converging subsequence. Of course, since $\left\{\tau^{[n]}: n \in \mathbb{N}\right\} \subseteq[0,1]$, $\left(\tau^{[n]}\right)_{n \in \mathbb{N}}$ converges to a certain $\bar{\tau} \in[0,1]$.

In order to prove the convergence of the sequence $\left(x^{[n]}\right)_{n \in \mathbb{N}}$, let us notice at first that, defined,

$$
\forall h \in \mathcal{H}, n \in \mathbb{N}, \bar{e}_{h}^{[n]}=\left(1-\tau^{[n]}\right) e_{h}+\tau^{[n]} x_{h}^{*} \in \mathbb{R}_{++}^{G} \quad \text { and } \quad \bar{e}_{h}=(1-\bar{\tau}) e_{h}+\bar{\tau} x_{h}^{*} \in \mathbb{R}_{++}^{G},
$$

we have that, for every $h \in \mathcal{H}, \bar{e}_{h}^{[n]} \rightarrow \bar{e}_{h}$. In particular, there exists $v_{h} \in \mathbb{R}_{++}^{G}$ such that, for every $n \in \mathbb{N}$, $\bar{e}_{h}^{[n]} \leq v_{h}$.

Fix now $h \in \mathcal{H}$. Since, for every $n \in \mathbb{N}, x_{h}^{[n]} \in \mathbb{R}_{++}^{G}$ and (20) holds, we have

$$
\left\{x_{h}^{[n]}: n \in \mathbb{N}\right\} \subseteq\left\{x_{h} \in \mathbb{R}^{G}: 0 \leq x_{h} \leq \sum_{h=1}^{H} v_{h}\right\}=C_{h}^{1} .
$$

By (19), for every $n \in \mathbb{N}$, we have

$$
u_{h}\left(x_{h}^{[n]}\right) \geq u_{h}\left(\bar{e}_{h}^{[n]}\right) \geq \min _{x_{h} \in\left\{\bar{e}_{h}^{[n]}: n \in \mathbb{N}\right\} \cup\left\{\bar{e}_{h}\right\}} u_{h}\left(x_{h}\right)=u_{h}\left(\underline{x}_{h}\right),
$$

where $\underline{x}_{h}$ is a suitable element of the compact set $\left\{\bar{e}_{h}^{[n]}: n \in \mathbb{N}\right\} \cup\left\{\bar{e}_{h}\right\} \subseteq \mathbb{R}_{++}^{G}$, and then

$$
\left\{x_{h}^{[n]}: n \in \mathbb{N}\right\} \subseteq\left\{x_{h} \in \mathbb{R}_{++}^{G}: u_{h}\left(x_{h}\right) \geq u_{h}\left(\underline{x}_{h}\right)\right\}=C_{h}^{2} .
$$

From (4), $C_{h}^{2}$ is a closed subset of $\mathbb{R}^{G}$, and then $C_{h}^{1} \cap C_{h}^{2}$ is a compact set contained in $\mathbb{R}_{++}^{G}$. As $\left\{x_{h}^{[n]}: n \in \mathbb{N}\right\} \subseteq C_{h}^{1} \cap C_{h}^{2}$, we have that $\left(x_{h}^{[n]}\right)_{n \in \mathbb{N}}$ converges to an element of $\mathbb{R}_{++}^{G}$, say $\bar{x}_{h}$, and then the convergence of $\left(x^{[n]}\right)_{n \in \mathbb{N}}$ to an element of $\mathbb{R}_{++}^{G H}$ is proved. From (18.1), (18.7) and (2) we find that, for every $h \in \mathcal{H}, s \in \mathcal{S}$,

$$
\lambda_{h}^{[n]}(s)=D_{x_{h}^{C}(s)} u_{h}\left(x_{h}^{[n]}\right) \rightarrow D_{x_{h}^{C}(s)} u_{h}\left(\bar{x}_{h}\right)=\bar{\lambda}_{h}(s) \in \mathbb{R}_{++},
$$

and, from (18.1) and (2), it follows, for every $s \in \mathcal{S}$,

$$
p^{[n]}(s)=\frac{D_{x_{h}(s)} u_{h}\left(x_{h}^{[n]}\right)}{\lambda_{h}^{[n]}(s)} \rightarrow \frac{D_{x_{h}(s)} u_{h}\left(\bar{x}_{h}\right)}{\bar{\lambda}_{h}(s)}=\bar{p}(s) \in \mathbb{R}_{++}^{C} .
$$

Then $\left(\lambda^{[n]}\right)_{n \in \mathbb{N}}$ converges to an element of $\mathbb{R}_{++}^{(S+1) H}$ and $\left(p^{[n]}\right)_{n \in \mathbb{N}}$ converges to an element of $\mathbb{R}_{++}^{G}$.

Fix now $a \in \mathcal{A}$ and consider the sequence $\left(q^{a,[n]}\right)_{n \in \mathbb{N}}$. We claim that it converges if there exist $h \in \mathcal{H}$ and a sequence $\left(n_{k}\right)_{k \in \mathbb{N}}$ in $\mathbb{N}$ such that, for every $k \in \mathbb{N}$,

$$
z_{h}^{a,\left[n_{k}\right]}+\left(1-\tau^{\left[n_{k}\right]}\right) \sigma_{h}^{a}\left(p^{\left[n_{k}\right]}, q^{\left[n_{k}\right]}\right)+\tau^{\left[n_{k}\right]} \varepsilon>0 .
$$

Indeed, if this is true, from (18.3) and (18.4) we get

$$
q^{a,\left[n_{k}\right]}=\frac{1}{\lambda_{h}^{\left[n_{k}\right]}(0)} \sum_{s=1}^{S} \lambda_{h}^{\left[n_{k}\right]}(s) p^{\left[n_{k}\right]}(s) y^{a}(s) \rightarrow \frac{1}{\bar{\lambda}_{h}(0)} \sum_{s=1}^{S} \bar{\lambda}_{h}(s) \bar{p}(s) y^{a}(s)=\bar{q}^{a} .
$$

In order to show the claim, assume by contradiction that there exists $\nu \in \mathbb{N}$ such that, for every $h \in \mathcal{H}$, $n \geq \nu$,

$$
z_{h}^{a,[n]}+\left(1-\tau^{[n]}\right) \sigma_{h}^{a}\left(p^{[n]}, q^{[n]}\right)+\tau^{[n]} \varepsilon=0 .
$$


Summing up on $h \in \mathcal{H}$, and using (18.6), we find

$$
0=\sum_{h=1}^{H} z_{h}^{a,[n]}+\left(1-\tau^{[n]}\right) \sum_{h=1}^{H} \sigma_{h}^{a}\left(p^{[n]}, q^{[n]}\right)+\tau^{[n]} H \varepsilon=\left(1-\tau^{[n]}\right) \sum_{h=1}^{H} \sigma_{h}^{a}\left(p^{[n]}, q^{[n]}\right)+\tau^{[n]} H \varepsilon .
$$

From (7), the right hand side of the above equality has to be positive. Then the contradiction is found and the convergence of $\left(q^{[n]}\right)_{n \in \mathbb{N}}$ to an element of $\mathbb{R}^{A}$ follows. Fix now $h \in \mathcal{H}, a \in \mathcal{A}$ and consider the sequence $\left(z_{h}^{a,[n]}\right)_{n \in \mathbb{N}}$. For every $n \in \mathbb{N}$, we have

$$
-\left(1-\tau^{[n]}\right) \sigma_{h}^{a}\left(p^{[n]}, q^{[n]}\right)-\tau^{[n]} \varepsilon \leq z_{h}^{a,[n]}=-\sum_{h^{\prime} \in \mathcal{H}, h^{\prime} \neq h} z_{h^{\prime}}^{a,[n]} \leq \sum_{h^{\prime} \in \mathcal{H}, h^{\prime} \neq h}\left(\left(1-\tau^{[n]}\right) \sigma_{h}^{a}\left(p^{[n]}, q^{[n]}\right)+\tau^{[n]} \varepsilon\right),
$$

and, since $\tau^{[n]} \rightarrow \bar{\tau}, p^{[n]} \rightarrow \bar{p}, q^{[n]} \rightarrow \bar{q}$ and (5) holds true, there exist $\underline{K}_{h}^{a}, \bar{K}_{h}^{a} \in \mathbb{R}$ such that, for every $n \in \mathbb{N}$,

$$
\underline{K}_{h}^{a} \leq z_{h}^{a,[n]} \leq \bar{K}_{h}^{a}
$$

Thus $z_{h}^{a,[n]} \rightarrow \bar{z}_{h}^{a}$ and the convergence of $\left(z^{[n]}\right)_{n \in \mathbb{N}}$ to an element of $\mathbb{R}^{A H}$ is proved. Finally, fix $h \in \mathcal{H}$, $a \in \mathcal{A}$ and study the convergence of the sequence $\left(\mu_{h}^{a,[n]}\right)_{n \in \mathbb{N}}$. From (18.3) we immediately obtain

$$
\mu_{h}^{a,[n]}=\lambda_{h}^{[n]}(0) q^{a,[n]}-\sum_{s=1}^{S} \lambda_{h}^{[n]}(s) p^{[n]}(s) y^{a}(s) \rightarrow \bar{\lambda}_{h}(0) \bar{q}^{a}-\sum_{s=1}^{S} \bar{\lambda}_{h}(s) \bar{p}(s) y^{a}(s)=\bar{\mu}_{h}^{a},
$$

and the convergence of $\left(\mu^{[n]}\right)_{n \in \mathbb{N}}$ to an element of $\mathbb{R}^{A H}$ is proved. Then the proof of (23) is complete.

We finally present the proof of Theorem 3 via Theorem 4.

Proof of Theorem 3. Let us consider at first the properties of $\mathcal{Y}_{2}$. If $A>C$, then we immediately have $\mathcal{Y}_{2}=\varnothing$. Assume then $A \leq C$. The fact that $\mathcal{Y}_{2}$ is open can be proved as follows. Observe that

$$
\mathcal{Y}_{2}=\bigcup_{\mathcal{K} \subseteq\{1, \ldots, S\},|\mathcal{K}|=A} \mathcal{Y}_{2}(\mathcal{K})
$$

where, for every $\mathcal{K} \subseteq\{1, \ldots, S\}$ such that $|\mathcal{K}|=A$,

$$
\mathcal{Y}_{2}(\mathcal{K})=\left\{y \in \mathbb{R}^{C A S}: \forall s \in \mathcal{K}, \operatorname{rank}\left(\left[y^{a, c}(s)\right]_{c \in \mathcal{C}, a \in \mathcal{A}}\right)=A\right\} .
$$

Note that, for every $\mathcal{K} \subseteq\{1, \ldots, S\}$ such that $|\mathcal{K}|=A$, the following equality holds

$$
\mathbb{R}^{C A S} \backslash \mathcal{Y}_{2}(\mathcal{K})=\bigcup_{s \in \mathcal{K}}\left(\bigcap_{\mathcal{I} \subseteq \mathcal{C},|\mathcal{I}|=A}\left\{y \in \mathbb{R}^{C A S}: \operatorname{det}\left(\left[y^{c, a}(s)\right]_{c \in \mathcal{I}, a \in \mathcal{A}}\right)=0\right\}\right) .
$$

As the right hand side of (35) is a closed subset of $\mathbb{R}^{C A S}$, it follows that $\mathcal{Y}_{2}(\mathcal{K})$ is an open subset of $\mathbb{R}^{C A S}$ and then, from (34), $\mathcal{Y}_{2}$ is an open subset of $\mathbb{R}^{C A S}$, as well.

In order to prove that $\mathcal{Y}_{2}$ is a full measure subset of $\mathbb{R}^{C A S}$, it is sufficient to show that the subset of $\mathcal{Y}_{2}$ defined as

$$
\mathcal{Y}_{3}=\left\{y \in \mathbb{R}^{C A S}: \forall s \in\{1, \ldots, S\}, \operatorname{rank}\left(\left[y^{a, c}(s)\right]_{c \in \mathcal{C}, a \in \mathcal{A}}\right)=A\right\}
$$

is a full measure subset of $\mathbb{R}^{C A S}$. To this end, we only need to show that, for every $s \in\{1, \ldots, S\}, 0$ is a regular value for the map $\Psi^{s}: \mathbb{R}^{A} \times \mathbb{R}^{C A S} \rightarrow \mathbb{R}^{C+1}$, defined as

$$
\Psi^{s}(v, y)=\left[\begin{array}{ll}
{\left[y^{a, c}(s)\right]_{c \in \mathcal{C}, a \in \mathcal{A}} v} & \frac{1}{2} v \cdot v-1 \\
\frac{1}{2} v \cdot v-1
\end{array}\right]
$$


In fact, if that is true, from Theorem 4 we have that, for every $s \in\{1, \ldots, S\}$, there exists a full measure subset $\mathcal{Y}_{3}(s)$ of $\mathbb{R}^{C A S}$ such that, for every $y \in \mathcal{Y}_{3}(s), 0$ is a regular value for the function $\Psi_{y}^{s}: \mathbb{R}^{A} \rightarrow \mathbb{R}^{C+1}$, $v \mapsto \Psi^{s}(v, y)$. Since $A<C+1$, that is equivalent to say that, for every $y \in \mathcal{Y}_{3}(s)$,

$$
\left\{v \in \mathbb{R}^{A}: \Psi^{s}(v, y)=0\right\}=\varnothing,
$$

that is,

$$
\operatorname{rank}\left(\left[y^{a, c}(s)\right]_{c \in \mathcal{C}, a \in \mathcal{A}}\right)=A \text {. }
$$

As $\cap_{s=1}^{S} \mathcal{Y}_{3}(s) \subseteq \mathcal{Y}_{3}, \mathcal{Y}_{3}$ has full measure in $\mathbb{R}^{C A S}$. Thus we are left with proving that, for every $s \in$ $\{1, \ldots, S\}, 0$ is a regular value for $\Psi^{s}$, that is, if $(v, y) \in \mathbb{R}^{A} \times \mathbb{R}^{C A S}$ satisfies $\Psi^{s}(v, y)=0$, then $D \Psi^{s}(v, y)$ has full rank. This fact immediately follows by the structure of $D \Psi^{s}(v, y)$.

Let us prove now the inclusion $\mathbb{R}_{++}^{G H} \times \mathcal{U} \times \mathcal{Y}_{1} \times \Sigma \subseteq \mathcal{E}^{\diamond}$. Consider then $(e, u, y, \sigma) \in \mathbb{R}_{++}^{G H} \times \mathcal{U} \times \mathcal{Y}_{1} \times \Sigma$ and let $\mathcal{K} \subseteq\{1, \ldots, S\}$, with $|\mathcal{K}|=A$, and $\alpha:\{1, \ldots, S\} \rightarrow \mathcal{C}$ such that (12) and (13) hold. As $\mathcal{P}(u) \neq \varnothing$ and, for every $x_{1} \in \mathbb{R}_{++}^{G}$,

$$
\operatorname{rank}\left(\mathcal{R}\left(D u_{1}\left(x_{1}\right), y\right)\right)=\operatorname{rank}\left(\left[y^{a, \alpha(s)}(s)\right]_{s \in \mathcal{K}, a \in \mathcal{A}}\right)=A,
$$

it immediately follows that $(e, u, y, \sigma) \in \mathcal{E}^{\diamond}$.

Finally, let us show the inclusion $\mathbb{R}_{++}^{G H} \times \mathcal{U} \times \mathcal{Y}_{2} \times \Sigma \subseteq \mathcal{E}^{\diamond}$. This is obvious if $A>C$, as $\mathcal{Y}_{2}=\varnothing$. Assume then $A \leq C$, fix $(e, u, y, \sigma) \in \mathbb{R}_{++}^{G H} \times \mathcal{U} \times \mathcal{Y}_{2} \times \Sigma$ and prove it belongs to $\mathcal{E}^{\diamond}$. First of all, let us recall that, given $(r, \underline{u})=\left(r, \underline{u}_{2}, \ldots, \underline{u}_{H}\right) \in \mathbb{R}_{++}^{G} \times \mathbb{R}^{H-1}$, if the maximization problem

$$
\max _{x \in \mathbb{R}_{++}^{G H}} u_{1}\left(x_{1}\right) \quad \text { s.t. } \quad\left\{\begin{array}{l}
u_{h}\left(x_{h}\right)=\underline{u}_{h}, \quad h \geq 2 \\
\sum_{h=1}^{H} x_{h}=r
\end{array}\right.
$$

has a feasible solution, then it has a unique optimal solution $x^{*}$ which is Pareto Optimal for $u$ and there exists $\left(\theta^{*}, \gamma^{*}\right) \in \mathbb{R}^{H} \times \mathbb{R}^{G}$ such that $\left(x^{*}, \theta^{*}, \gamma^{*}\right)$ solves the system

$$
\left\{\begin{array}{l}
\theta_{h} D u_{h}\left(x_{h}\right)-\gamma=0 \\
u_{h}\left(x_{h}\right)-\underline{u}_{h}=0, \quad h \geq 2 \\
\theta_{1}-1=0 \\
-\sum_{h=1}^{H} x_{h}+r=0
\end{array}\right.
$$

Conversely, if there exists $\left(x^{*}, \theta^{*}, \gamma^{*}\right)$ solving System (37), then $x^{*}$ is the unique optimal solution to Problem (36) and it is a Pareto Optimal allocation for $u$. Consider then $\mathcal{K} \subseteq\{1, \ldots, S\}$, with $|\mathcal{K}|=A$, such that (14) holds and define the function

$$
\begin{gathered}
\Phi: \mathbb{R}_{++}^{G H} \times \mathbb{R}^{H} \times \mathbb{R}^{G} \times \mathbb{R}^{A} \times \mathbb{R}_{++}^{G} \times \mathbb{R}^{H-1} \rightarrow \mathbb{R}^{G H+H+G+A+1} \\
\Phi(x, \theta, \gamma, v, r, \underline{u})=\left[\begin{array}{lll}
(38.1) & \theta_{1} D u_{1}\left(x_{1}\right)-\gamma & \\
(38.2) & \theta_{1}-1 \\
(38.3) & \theta_{h} D u_{h}\left(x_{h}\right)-\gamma, & h \geq 2 \\
(38.4) & u_{h}\left(x_{h}\right)-\underline{u}_{h}, & h \geq 2 \\
(38.5) & -\sum_{h=1}^{H} x_{h}+r & \\
(38.6) & \mathcal{R}_{\mathcal{K}}(\gamma, y) v & \\
(38.7) & \frac{1}{2} v v-1
\end{array}\right]
\end{gathered}
$$


where $\mathcal{R}_{\mathcal{K}}(\gamma, y)$ is the matrix built by considering only the rows of $\mathcal{R}(\gamma, y)$ whose index is in $\mathcal{K}$. Of course $\mathcal{R}_{\mathcal{K}}(\gamma, y) \in \mathbb{M}(A, A)$. Let us prove now that 0 is a regular value of $\Phi$.

The Jacobian of $\Phi$ is described by the table below, where $h \geq 2$. The rank computation is performed through row and column operations. The starred matrices are full row rank matrices that we employ to "clean up" its super-row, since all the other elements of its super-column are null. A suitable order in which the appropriate elementary super-column operations have to be performed is the one indicated in the last column of the table.

\begin{tabular}{c||c|c|c|c|c|c|c|c||c|}
\cline { 2 - 9 } \multicolumn{1}{c|}{} & $x_{1}$ & $\theta_{1}$ & $x_{h}$ & $\theta_{h}$ & $\underline{u}_{h}$ & $r$ & $\gamma$ & $v$ & \\
\hline \hline$(38.1)$ & $\theta_{1} D^{2} u_{1}\left(x_{1}\right) *$ & $D u_{1}\left(x_{1}\right)$ & & & & & $-I_{G}$ & & 2 \\
\hline$(38.2)$ & & $1 *$ & & & & & & & 3 \\
\hline$(38.3)$ & & & $\theta_{h} D^{2} u_{h}\left(x_{h}\right) *$ & $D u_{h}\left(x_{h}\right)$ & & & $-I_{G}$ & & 2 \\
\hline$(38.4)$ & & & $D u_{h}\left(x_{h}\right)$ & & $-1 *$ & & & & 3 \\
\hline$(38.5)$ & $-I_{G}$ & & $-I_{G}$ & & & $I_{G} *$ & & & 1 \\
\hline$(38.6)$ & & & & & & & $M(y, v) *$ & $\mathcal{R}_{\mathcal{K}}(\gamma, y)$ & 4 \\
\hline$(38.7)$ & & & & & & & & $v *$ & 5 \\
\hline
\end{tabular}

Of course, we are left with showing that the matrix $M(y, v)$ indeed has full row rank. If we write

$$
M(y, v)=\left[\left(m_{s^{\prime}}^{0}(y, v), m_{s^{\prime}}^{1}(y, v), \ldots, m_{s^{\prime}}^{S}(y, v)\right)\right]_{s^{\prime} \in \mathcal{K}} \in \mathbb{M}(A, G),
$$

where

$$
\begin{array}{ll}
\forall s^{\prime} \in \mathcal{K}, s \in \mathcal{S}, & m_{s^{\prime}}^{s}(y, v) \in \mathbb{R}^{C}, \\
\forall s^{\prime} \in \mathcal{K}, & \left(m_{s^{\prime}}^{0}(y, v), m_{s^{\prime}}^{1}(y, v), \ldots, m_{s^{\prime}}^{S}(y, v)\right) \in \mathbb{R}^{G},
\end{array}
$$

then a simple computation shows that

$$
m_{s^{\prime}}^{s}(y, v)= \begin{cases}\sum_{a \in \mathcal{A}} y^{a}\left(s^{\prime}\right) v^{a} & \text { if } s^{\prime}=s \\ 0 & \text { if } s^{\prime} \neq s\end{cases}
$$

Then, using Assumption (14), the full row rank property of $M(y, v)$ immediately follows.

Applying now Theorem 4 , we find there exists a full measure subset $\mathcal{D}$ of $\mathbb{R}_{++}^{G} \times \mathbb{R}^{H-1}$ such that, for every $(r, \underline{u}) \in \mathcal{D}, 0$ is a regular value of the function

$$
\Phi_{(r, \underline{u})}: \mathbb{R}_{++}^{G H} \times \mathbb{R}^{H} \times \mathbb{R}^{G} \times \mathbb{R}^{A} \rightarrow \mathbb{R}^{G H+H+G+A+1}, \quad(x, \theta, \gamma, v) \mapsto \Phi(x, \theta, \gamma, v, r, \underline{u}) .
$$

As a consequence, for every $(r, \underline{u}) \in \mathcal{D}$,

$$
\{(x, \theta, \gamma, v): \Phi(x, \theta, \gamma, v, r, \underline{u})=0\}=\varnothing .
$$

From the above considerations, we are done if we prove that $\Psi\left(\mathbb{R}_{++}^{G H}\right) \cap \mathcal{D} \neq \varnothing$, where

$$
\Psi: \mathbb{R}_{++}^{G H} \rightarrow \mathbb{R}_{++}^{G} \times \mathbb{R}^{H-1}, \quad x \mapsto\left(\sum_{h=1}^{H} x_{h},\left(u_{h}\left(x_{h}\right)\right)_{h=2}^{H}\right) .
$$

It is immediate to prove that, for every $x \in \mathbb{R}_{++}^{G H}, D \Psi(x)$ is surjective. Then $\Psi\left(\mathbb{R}_{++}^{G H}\right)$ is an open subset of $\mathbb{R}_{++}^{G} \times \mathbb{R}^{H-1}$ and indeed $\Psi\left(\mathbb{R}_{++}^{G H}\right) \cap \mathcal{D} \neq \varnothing$.

\section{References}

Araujo, A., Orrillo, J. and M.R. Pascoa, (2000), Equilibrium with default and endogenous collateral, Mathematical Finance; 10; 1-21.

Bisin, A., Geanakoplos, J., Gottardi, P., Minelli E. and H. Polemarchakis, (2010), Markets and contracts, preprint. 
Bottazzi, J.-M., (1995), Existence of equilibria with incomplete markets: the case of smooth returns, Journal of Mathematical Economics; 24; 59-72.

Carosi, L., Gori, M. and A. Villanacci, (2009), Endogenous restricted participation in general financial equilibrium, Journal of Mathematical Economics; 45; 787-806.

Debreu, G., (1959), Theory of value: an axiomatic analysis of economic equilibrium, Cowles Foundation Monograph 17, John Wiley \& Sons, Inc., New York.

Dubey, P., Geanakoplos, J. and M. Shubik, (2005), Default and punishment in general equilibrium, Econometrica; 73; 1-37.

Duffie, D. and W. Shafer, (1985), Equilibrium with incomplete markets: I. A basic model of generic existence, Journal of Mathematical Economics; 14; 285-300.

Geanakoplos, J., (1997), Promises promises, in W.B. Arthur, S. Durlauf and D. Lane (eds.), The Economy as an Evolving Complex System, II. Reading MA: Addison-Wesley; 285-320.

Geanakoplos, J. and W. Shafer, (1990), Solving a system of simultaneous equations in economics, Journal of Mathematical Economics; 19; 69-95.

Hirsch, M.D., Magill, M. and A. Mas-Colell, (1990), A geometric approach to a class of equilibrium existence theorems, Journal of Mathematical Economics; 19; 95-107.

Husseini, S., Lasry, J.-M. and M. Magill, (1990), Existence of equilibrium with incomplete markets, Journal of Mathematical Economics; 19; 39-68.

Radner, R., (1972), Existence of equilibrium of plans, prices, and price expectations in a sequence of markets, Econometrica; 40; 289-303.

Seghir, A. and J.P. Torres-Martinez, (2011), On equilibrium existence with endogenous restricted financial participation, Journal of Mathematical Economics; 47; 37-42.

Villanacci, A., Carosi, L., Benevieri, P. and A. Battinelli, (2002), Differential topology and general equilibrium with complete and incomplete markets, Kluwer Academic Publishers. 